\title{
Comparing Surface Chemical Modifications of Zinc Oxide Nanoparticles for Modulating their Antiviral Activity against Herpes Simplex Virus Type-1
}

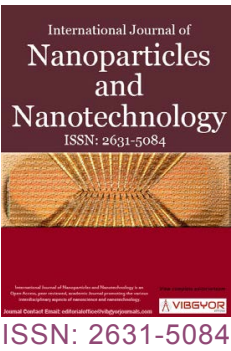

Faten Farouk ${ }^{1 \star}$ and Rania Ibrahim Sheb/ ${ }^{2}$

${ }^{1}$ Pharmaceutical Chemistry Department, Faculty of Pharmacy, Ahram Canadian University, Cairo, Egypt

${ }^{2}$ Microbiology and Immunology Department, Faculty of Pharmacy, Ahram Canadian University, Cairo, Egypt

\begin{abstract}
Herpes-simplex virus type-1 (HSV-1) represents a life persistent infection. It is primarily treated by acyclovir however resistant strains are increasing which urged the development of new treatment strategies. Zinc nanoparticles (ZNPs) based therapeutics is gaining a lot of attention. The current study aims to investigate variable physicochemical interactions of ZNPs bearing different chemical moieties and surface properties with the cell receptors and the viral particles, and their effect on reducing HSV-1 infection.
\end{abstract}

ZNPs were selected as a scaffold for anchoring active moieties designed against HSV-1. Hydroxyl group rich ZNPs (H-ZNPs), oleic acid modified ZNPs (OA-ZNPs) and chitosan-Zinc nanoparticles (C-ZNPs) were chemically synthesized and their characterization was performed by ultra-violet (UV) and Fourier transform infra-red (FTIR) spectroscopy, transmission electron microscope (TEM) and selected area electron diffraction (SAED). Vero cells were pretreated with ZNPs prior to viral infection to determine the ability of the particles to interact with cellular targets. HSV-1 was treated with ZNPS for different intervals to evaluate their viral neutralization kinetics. The ability of ZNPs to block the HSV-1 infection was compared in both cases.

Inoculating the ZNPs with HSV-1 was more effective than the cellular pretreatment in reducing the viral infectivity; where H-ZNPs and OA-ZNPs resulted in reducing the viral infectivity titer in the order of $12.9 \%$ and $7.7 \%$; respectively in nanoparticles pretreated cells whereas C-ZNPs were ineffective. On the other hand, incubating HSV-1 with H-ZNPs and C-ZNPs showed a greater time dependent inhibition and completely inactivated the virus within a period of $24 \mathrm{~h}$ while OA-ZNPs exhibited the lowest (15\%) reduction in the viral titer.

These results suggest that surface modified ZNPs could modify the HSV-1 infectivity potential via neutralizing the virus rather than through interfering with cellular targets. In this respect the physical entrapment exerted by C-ZNPs and the electrostatic interference of H-ZNPs are more significant than the hydrophobic interaction using OA-ZNPs.

\section{Keywords}

Zinc oxide nanoparticles, Herpes-simplex virus type-1, Antiviral agents, Nano-particles based therapeutics

*Corresponding author: Faten Farouk, PhD, Assistant Professor of Pharmaceutical Chemistry, Faculty of Pharmacy, Ahram Canadian University, Cairo, Egypt, Tel: 01223308082

Accepted: December 27, 2018; Published: December 29, 2018

Copyright: (C) 2018 Farouk F, et al. This is an open-access article distributed under the terms of the Creative Commons Attribution License, which permits unrestricted use, distribution, and reproduction in any medium, provided the original author and source are credited. 


\section{Introduction}

Herpes simplex virus type-1 (HSV-1) infection is becoming a public health concern due to its wide-spread prevalence over the last decades [1]. According to the World Health Organization, an estimate of two-thirds of the population under 50 are infected with HSV-1 [2]. HSV-1 infections are common in oral and perioral area causing oral herpes or herpes labialis. It causes painful sores known as cold sores which are found on the upper and lower lips. It can also be extended on the tongue, roof of the mouth, sometimes on the face or even inside or on the nose. It may result in swollen lymph nodes or fever [3]. Infrequently,
HSV-1 may also cause genital lesions [4,5]. The clinical complications associated with HSV-1 may be exaggerated to serious cases such as corneal blindness and encephalitis [6].

Most people acquire primary HSV-1 infection in early childhood although the infection could occur at any age. Following the primary infection, the virus remains latent in the nerve cells and is present for life. The virus could be reactivated by physical or emotional stress, sunlight, another viral infection or hormonal changes. HSV-1 transmission takes place via personal contact and infection occurs by viral inoculation onto the mucosal membranes or through small cracks in the skin [7].

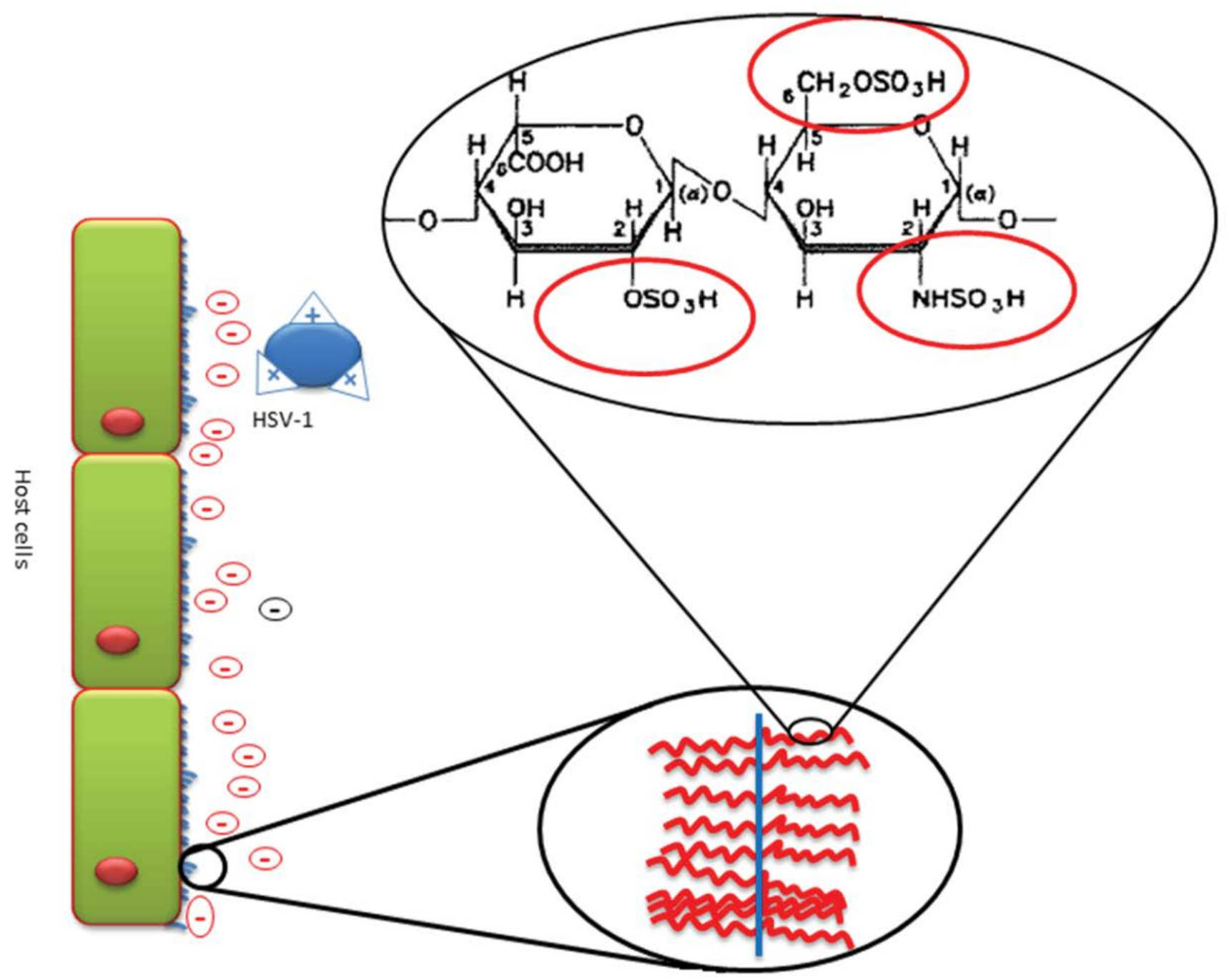

Polysaccharide chain attached to Peptidoglycan core

Figure 1: Illustration of the HS on the host cell surface. HS appear as a polysaccharide attached by the peptidoglycan core to the cell surface giving a negative charge on the cell surface. Closer looks on composition of the polysaccharide reveal the most repeated sulfated disaccharide of HS (GlcA linked to GIcN). The figure illustrates the HSV-1 having surface cationic groups enabling its electrostatic interaction with negatively charged sulfated regions of HS. 
HSV-1 is composed of double stranded DNA enclosed in a capsid. The capsid is in-turn surrounded by another layer called tegument. The viral components are all surrounded by a lipid bilayer decorated with a group of glycoproteins. Viral glycoproteins (gp) function in its attachment and fusion to cell membrane. Initial attachment is mediated through gp interaction with the heparan sulfate (HS) present on cell surface $[7,8]$.

HS is an anionic structure which is composed of a series of sulfated disaccharides subunits linked to form a complex and highly acidic polysaccharide. It belongs to the glycosaminoglycan family and is attached to cell surface by its peptidoglycan core exposing the Polysaccharide chain on cell surface and extracellular matrix [9]. The most common disaccharide in the HS structure is that composed of glucuronic acid (GlcA) linked to $\mathrm{N}$-acetyl glucosamine (GlcN). These disaccharide subunits are modified by the sulfation at the hydroxyl group of the GlcA and N-sulfonation of GICN after its deacylation [9]. The sulfated disaccharide exists in clusters on the polysaccharide chain separated by unsulfated units. The sulfation pattern vary according to cell type [10]. HS is expressed on most of the mammalian cells. It functions in regulating numerous cell activities including cell adhesion, proliferation and binding to proteins $[11,12]$. This is mediated by its unique sulfation pattern which offers hot-spots for binding and interaction of HS with various proteins (Figure 1) [13].

In a close fashion HS acts as a receptor for many viruses and bacteria [14]. Using the negativity of its sulfated regions it can readily interact with the positively charged glycoprotein B (gB) and glycoprotein $\mathrm{C}(\mathrm{gC})$ found on HSV-1 surface to facilitate the initial viral attachment to cell [8]. Specifically, the positively charged amino-acid sequence of gC (Arg-143, Arg-145, Arg-147, Thr-150 and Gly-247) and that of gB (lysine-rich sequence (residues 68 to 76)) were suggested to be essential for the initial electrostatic interaction of the virus with the 6-O- and 2-O-sulfate groups found on $\mathrm{HS}$ $[15,16]$.

In addition to the role of HS in the electrostatic attraction of virus, the sulfated $3-\mathrm{OH}$ position of the HS glucosamine represents a binding site for glycoprotein $D(g D)$ and contributes in the entry of HSV into the cell [14].

Following electrostatic interactions, viral fusion into cell membrane takes place. This is mediated by viral glycoproteins which have hydrophobic segment known as fusion peptide. The glycoproteins involved in fusion are folded so as to expose its hydrophobic segment enabling insertion into cell membrane lipid bilayer $[17,18]$. For example, glycoprotein $\mathrm{H}(\mathrm{gH})$ of HSV-1 has the peptide gH-(626644) which is rich in hydrophobic residues such as glycines, leucines, alanines, as well as tryptophan and tyrosines and has limited proportion of charged amino-acids. Such structure enables the interaction and fusion with membrane bilayer based on hydrophobicity. It was reported that, compromising the hydrophobicity of the $\mathrm{gH}$ region by residue mutation resulted in reduced viral ability to penetrate cell membrane which supports the role of this hydrophobic region in viral entry [17]. Similarly, is the Cys127-Cys144 loop of the gC N terminus [15].

Acyclovir and related drugs have been the gold standard for the treatment of HSV-1 [6]. However resistant strains are rising especially in immunocompromised patients. This poses a clinical risk in such population where the viral complications may be fatal. Accordingly, alternative therapeutic approaches are gaining great interest [6].

The promising role of zinc in combating viruses (including HSV) and the evolution in the nanoparticle synthesis and modification for addressing biological targets directed the researchers to investigate the factors affecting the antiviral potentials of zinc nanoparticles [19-21]. Previous reports proved the virostatic potential of the negatively charged zinc tetrapods against both HSV-1 and HSV-2 and attributed it to the electrostatic interaction with the positively charged glycoprotein present on viral envelope [22-24].

The current study aimed to investigate the impact of the viral entrapment and the interference with its hydrophobic interactions with cell menrance on reducing viral infectivity. Moreover, their impact was compared to that of electrostatic interference in terms of viral neutralization capabilities and inhibition of early stages of viral entry.

\section{Materials and Methods}

\section{Materials}

$\mathrm{ZnCl}_{2}$ and $\mathrm{NaOH}$ were purchased from Fischer scientific, USA. Xylene and oleic acid were obtained from Gomhoria for Chemical industries, Egypt. Vero cells (African green monkey kidney cells - ATCC No. 
CCl-181) were kindly supplied from cell culture department, VACSERA. HSV-1 was supplied from research and development unit, VACSERA virology sector.

\section{Methods}

Synthesis and characterization of $\mathrm{ZnO}$ structures: Hydroxyl group rich zinc oxide nanoparticles (H-ZNPs) were synthesized by the co-precipitation method with some modifications $[25,26]$. Briefly 1 gram of polyethylene glycol 6000 was dissolved in $10 \mathrm{~mL}$ of ethanol. On Stirring, $3 \mathrm{~mL}$ of $1 \mathrm{M} \mathrm{ZnCl}_{2}$ solution was added and stirring was continued for $30 \mathrm{~min}$. Then, $1 \mathrm{M} \mathrm{NaOH}$ were added drop-wise with stirring until the solution turned basic $(\mathrm{pH}$ 11.6). Particles were then collected by centrifugation and washed with ethanol.

Chitosan-zinc oxide composite particles (C-ZNPs) were prepared by dispersing $20 \mathrm{mg}$ of chitosan powder in $20 \mathrm{~mL}$ deionized water followed by the addition of $1 \mathrm{~mL}$ of glacial acetic acid. The now dissolved chitosan was left on stirring for at least $1 \mathrm{hr}$ followed by the addition of $6 \mathrm{~mL}$ of $1 \mathrm{M} \mathrm{ZnCl}_{2}$ solution. The mixture was left on stirrer overnight. $\mathrm{NaOH}(1 \mathrm{M})$ was added drop wise until the solution turned basic ( $\mathrm{pH} \mathrm{11).} \mathrm{The} \mathrm{formed} \mathrm{cloudy} \mathrm{solution}$ was left on stirrer overnight and then collected by centrifugation and washed with deionized water. Prepared particles were characterized by transmission electron microscope (TEM) and Fourier-transform infrared (FTIR) spectroscopy.

For preparation of oleic acid coated $\mathrm{ZnO}$ (OAZNP), $0.5 \mathrm{~g}$ of $\mathrm{H}-\mathrm{ZNP}$ were transferred into a round bottom flask and $1.5 \mathrm{~mL}$ of oleic acid were added. The mixture was dispersed in $50 \mathrm{~mL}$ of xylene and left under reflux with continuous stirring for $6 \mathrm{hr}$. The reaction mixture was then left at room temperature overnight and then the modified particles were separated by centrifugation. Prepared OAZNPs were then washed using ethanol.

$\mathrm{H}-Z N P$ s and C-ZNPs particles were directly dispersed in Minimum Essential Media supplemented with Earle's salt (E-MEM) (GIBCO-USA) aided by probe sonication, while OA-ZNPs particles were dispersed by adding $20 \mu \mathrm{L}$ of tween 80 . The effect of the prepared NPs on viral infectivity titer was evaluated.

Maintenance of Vero cell line: Vero cell line was cultured in E-MEM supplemented with $10 \%$ fetal bovine serum (FBS) (GIBCO-USA). Vero cells were sub-cultured by trypsinization and the prepared suspension was inoculated into fresh flasks. Sixtynine well cell culture plates were also inoculated with the prepared cell suspension as $100 \mu \mathrm{l} /$ well and incubated at $37^{\circ} \mathrm{C}$ till confluency [27].

Propagation of HSV-1: HSV-1 virus was inoculated as 0.5 multiplicity of infection into cell culture flasks $\left(75-\mathrm{cm}^{2}\right)$ cultured with Vero cells after removal of its culture media. Flasks were incubated at 37 ${ }^{\circ} \mathrm{C}$ for an adsorption period of 1-1.5 hr with gentle mixing at 15 min time interval. After the adsorption period, an additional amount of maintenance medium (supplemented with $2 \%$ FBS) was added to each flask without removing the inoculum. Negative cell culture control flask was also considered. Cultures were incubated at $37{ }^{\circ} \mathrm{C}$ and examined daily using inverted microscope (Hund-Germany) till the development of $90 \%$ cytopathic effect (CPE). Virus was extracted from culture media by three cycles of successive freezing and thawing. Extracted virus was cryo-preserved in aliquots of $1 \mathrm{~mL}$ at $-70{ }^{\circ} \mathrm{C}[28]$.

Cytotoxicity assay: Evaluation of the cytotoxic effect of the prepared ZNPs on Vero cells was carried out to determine the cell safe concentration of ZNPs. This is to ensure that any observed cytopathic effect is solely attributed to HSV-1. This was performed by using the 3-(4,5-dimethylthiazol-2-yl)-2,5-diphenyltetrazolium bromide (MTT) assay. Briefly, ninety-six-well plates pre-cultured with Vero cells were treated with double fold serially diluted ZNPs and incubated at $37^{\circ} \mathrm{C}$. Plates were washed with phosphate buffer saline (PBS) and inoculated with MTT dye $(0.5 \mathrm{mg} / \mathrm{mL})$ as $100 \mu \mathrm{l} /$ well then incubated at $37^{\circ} \mathrm{C}$ for $4 \mathrm{hrs}$. At the end of the incubation period, MTT was discarded and plates were PBS washed three times. The formazan blue crystals that were produced in the viable cells were dissolved by adding dimethyl sulfoxide (DMSO) as $50 \mu \mathrm{l} /$ well followed by shaking the plates on plate shaker for $30 \mathrm{~min}$. Optical densities (ODs) were measured at $570 \mathrm{~nm}$ using ELISA plate reader (Dynatech-England). Percentages of cellular viability of three independent experiments were calculated according to the following equation: Cellular viability $\%=$ (absorbance of treated cells/absorbance of untreated cells) $\times 100$ [29]. The observed non-toxic concentrations "cell-safe concentrations" were subsequently used to assess the effect of the prepared ZNPs on the early stages of HSV infection and their viral inhibitory potential. 
Effect on early stages of viral infection: The ability of the tested NPs to interfere with early stages of viral replication was determined by treating Vero cells pre-cultured 96-well plates with the non-cytotoxic concentrations of the prepared ZNPs for $24 \mathrm{hr}$ at $37{ }^{\circ} \mathrm{C}$ which were previously determined using cytotoxicity test to be $0.0,0.1$ \& $0.24 \mathrm{mg} / \mathrm{mL}$ for OA-ZNPs, H-ZNPs \& C-ZNPs; respectively. Nanoparticles were discarded, plates were inoculated with tenfold serially diluted HSV-1 and incubated at $37^{\circ} \mathrm{C}$. Plates were examined daily using the inverted microscope till the development of CPE. Media were discarded, and plates were stained using $0.05 \%$ crystal violet in $3.7 \%$ formaldehyde for $30 \mathrm{~min}$ at room temperature. Stain was removed, and cells were rinsed with water and air dried. Viral titer was evaluated in nanoparticles treated and untreated cells by determining $\mathrm{TCID}_{50}$ (50\% tissue culture infectious dose of a virus) using Reed-Muench method [30].

Viral inhibitory potential: Evaluation of the viral inhibitory potential of the prepared nanoparticles was performed according to Ramadan, et al. [31], where the tested viral suspension was mixed with equal volume of non-toxic concentrations of ZNPs. Negative control virus was also considered by mixing it with nanoparticles free media. All sets were incubated at $37^{\circ} \mathrm{C}$ for $1,2,4$ and $24 \mathrm{hr}$. Zinc nanoparticles treated, and untreated virus was inoculated in 96-well Vero pre-cultured plates and the mean viral titers were determined as previous.

Statistical analysis: For all experiments, results were expressed as the mean \pm standard deviation of three independent tests. Statistical significance was determined using one-way analysis of variance (ANOVA). Differences at $P$ values less than 0.05 were considered statistically significant.
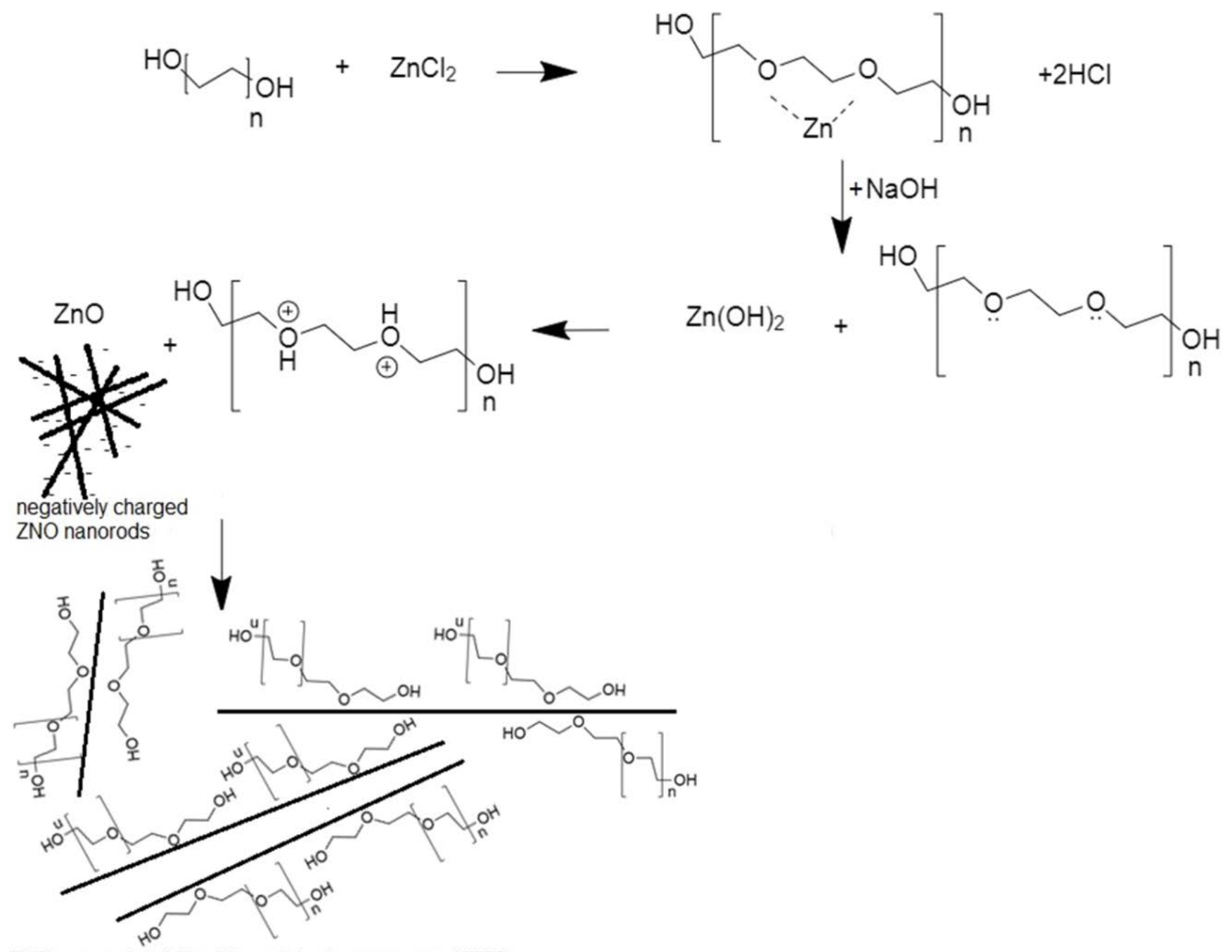

ZNO nanorod stabilized by poly hydroxy groups of PEG

Figure 2a: A proposed mechanism for the synthesis of H-ZNPs. 


\section{Results}

\section{Synthesis and characterization of zinc nano- structures}

Three zinc containing nanostructures were successfully synthesized. The first particle species was the hydroxyl-group rich ZNPs (H-ZNPs). Synthesis of these particles were performed in alcoholic medium to guard against particle agglomeration which may be induced by the formation of $\mathrm{Zn}-\mathrm{O}-\mathrm{Zn}$ bond [32]. In order to further stabilize the particles and enrich its surface with poly-hydroxyl groups the al-

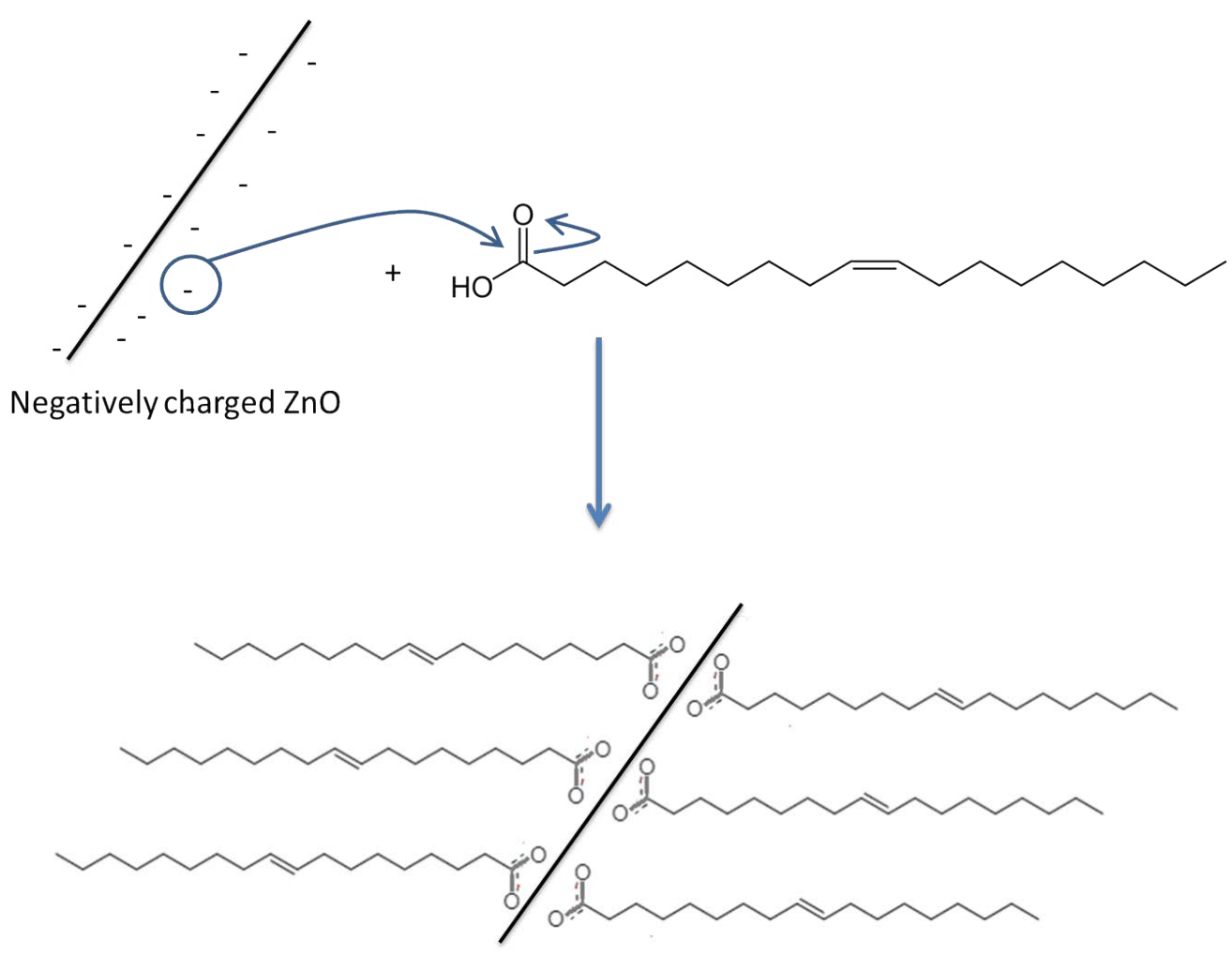

Figure $\mathbf{2 b}$ : A proposed mechanism for synthesis of OA-ZNPs.
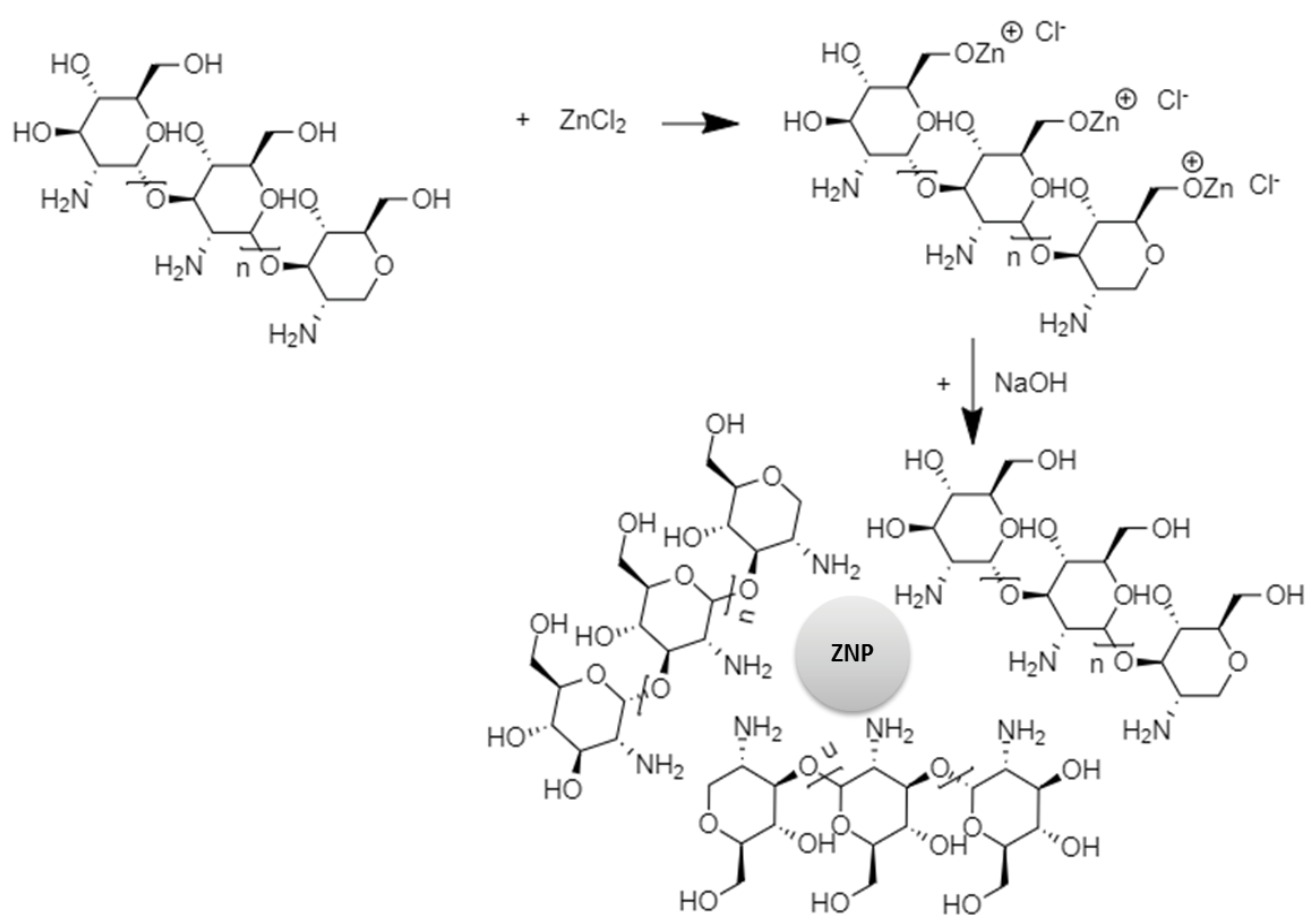

Figure 2c: A proposed mechanism for synthesis of C-ZNPs. 
coholic reaction medium was enriched with $10 \mathrm{~g} \%$ W/V PEG 600 (Figure 2a) [32,33].

The TEM image of the H-ZNPs showed that they were rod shaped with average width of $7 \mathrm{~nm}$ (Figure 3a). The formed H-ZNP were highly crystalline as evident from the selected area electron diffraction image (Figure 4a). The FTIR spectrum showed Zn-O stretching band at approximately $470 \mathrm{~cm}^{-1}$ [34]. The broad peak at $3475 \mathrm{~cm}^{-1}$ could be attributed to the stretching vibration of the $\mathrm{OH}$ groups while the 1637 is due to its bending vibration (Figure 5a). The
UV absorbance band revealed the formation of the H-ZNP (Supplementary Material).

Successful functionalization of the surface $\mathrm{OH}$ groups with oleic acid was achieved to give rise to the OA-ZNPs. A proposed chemical functionalization mechanism is presented in Figure $2 b$. The TEM image of the ZNPs after functionalization is presented in Figure $3 \mathrm{~b}$. The color of the ZNPs changed from white to yellowish white after the functionalization. Contrary to H-ZNPs, OA-ZNPs were indispensable in water. The FTIR spectrum of OA-ZNPs showed the

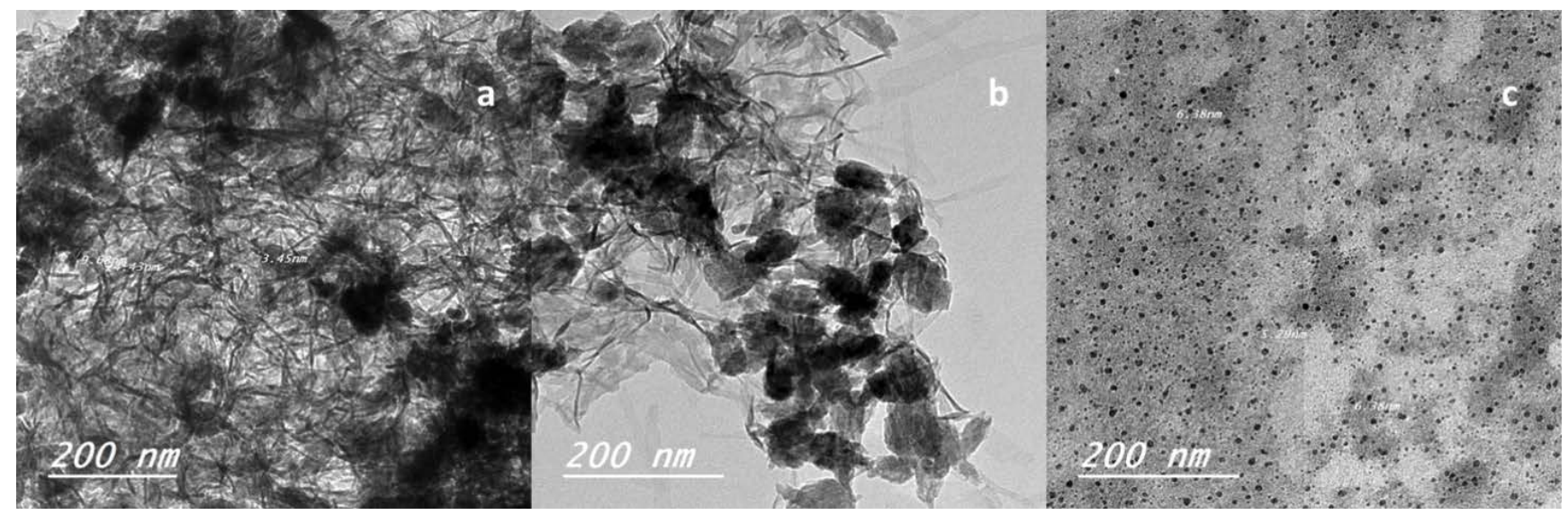

Figure 3: a) The TEM of synthesized H-ZNP. H-ZNPs appear rod shaped of average width $7 \mathrm{~nm}$; b) The TEM image of OA-ZNPs after functionalization; c) The TEM of C-ZNPs showing the ZNPs as spherical particles of an average size was $5 \mathrm{~nm}$ scattered in chitosan matrix.

Figure 4: Selected area electron diffraction pattern of the zinc nanorods (a; H-ZNPs and OA-ZNPs) and zinc nanoparticles (b; C-ZNPs). 


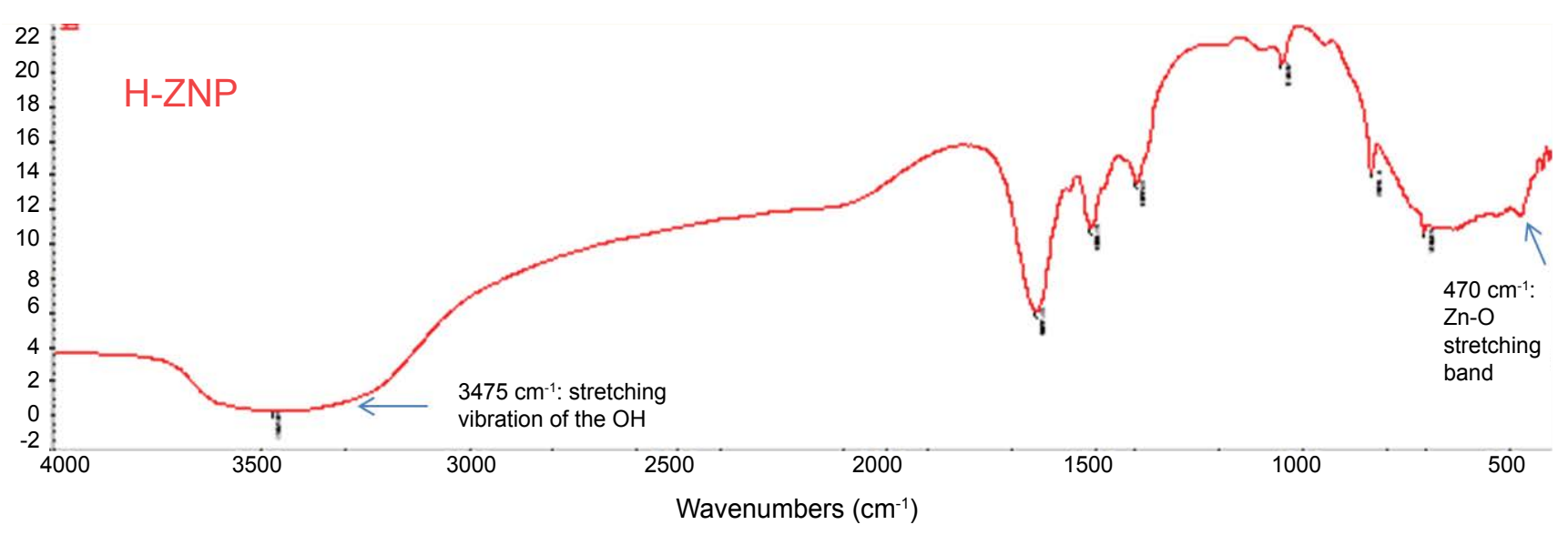

Figure 5a: FTIR spectra of H-ZNPs.

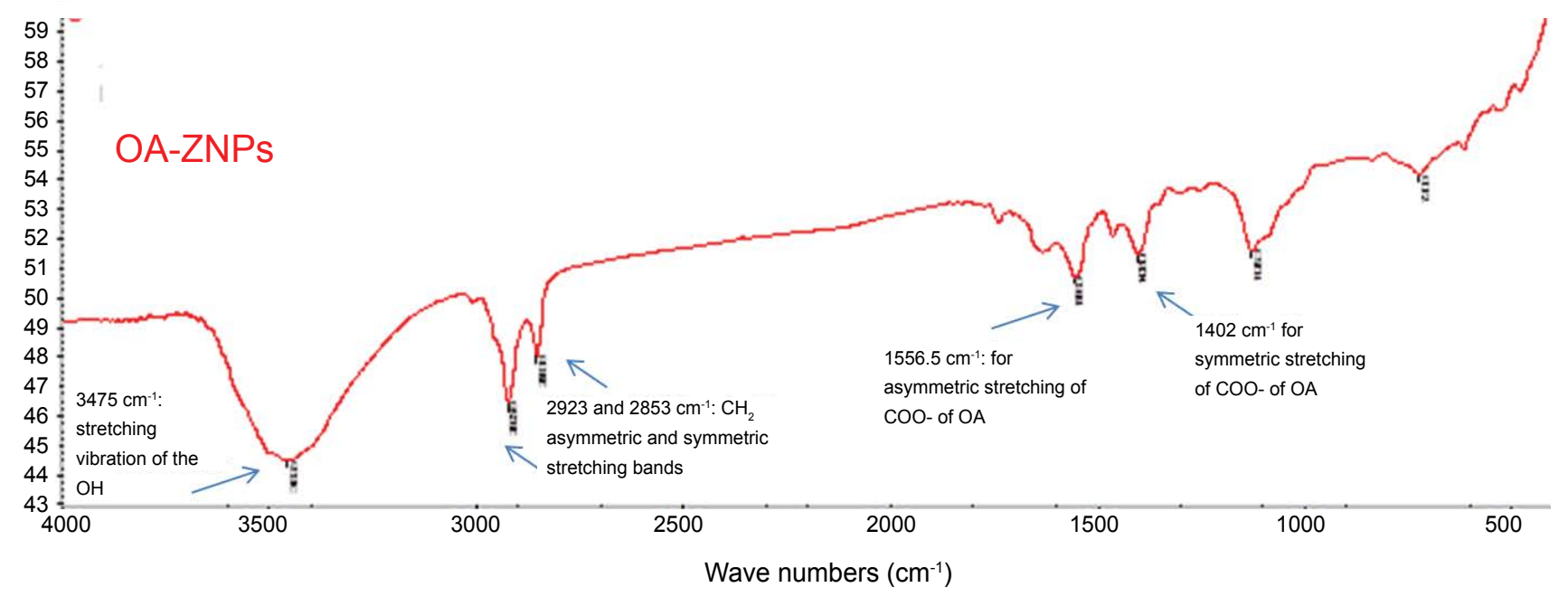

Figure 5b: FTIR spectra of OA-ZNPs.

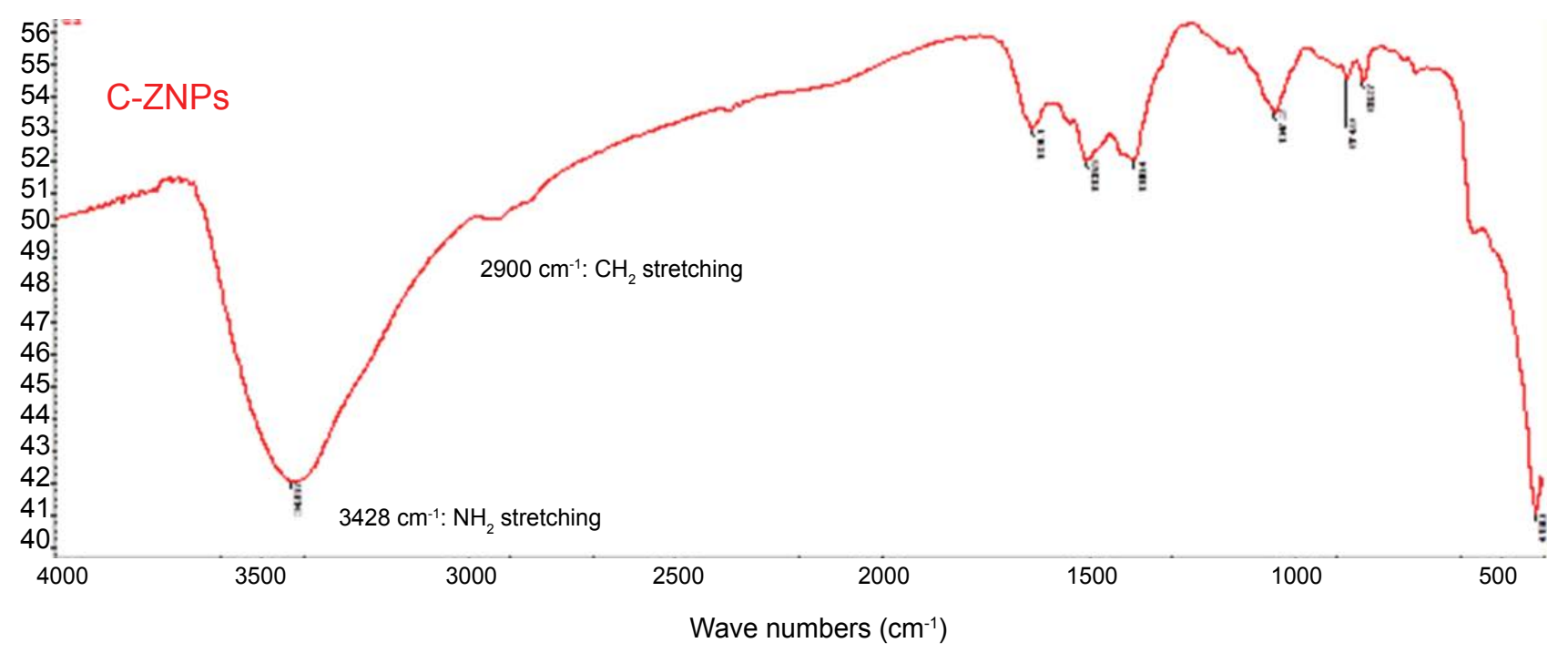

Figure 5c: FTIR spectra of C-ZNPs.

$\mathrm{CH}_{2}$ asymmetric and symmetric stretching bands of ly detected in the ZNPs was reduced probably due the long chain fatty acid at 2923 and $2853 \mathrm{~cm}^{-1}$, re- to its partial consumption in reaction with $\mathrm{COOH}$ of spectively. The broad OH band $\left(3475 \mathrm{~cm}^{-1}\right)$ previous- the OA. The COO- of OA appeared at $1556.5 \mathrm{~cm}^{-1} \mathrm{for}$ 
asymmetric stretching and $1402 \mathrm{~cm}^{-1}$ for symmetric stretching (Figure $5 \mathrm{~b}$ ). The difference between the two bands (approximately $150 \mathrm{~cm}^{-1}$ ) indicates that the binding is a bridged bi-dentate form [35]. The UV absorbance band of OA-ZNP is presented in the Supplementary Material.

C-ZNPs were successfully synthesized according to the prosed mechanism presented in Figure 2c. The morphology of C-ZNPs was distinctively different from the H-ZNPs. This was evident from the TEM which revealed the C-ZNPs as spherical particles of average diameter $5 \mathrm{~nm}$ embedded into chitosan matrix (Figure 3c). The electron diffraction of the synthesized C-ZNPs is shown in Figure $2 b$. The FTIR spectrum of the composite shows the chitosan $\mathrm{NH}_{2}$ band at $3428 \mathrm{~cm}^{-1}$ while the $\mathrm{CH}_{2}$ stretching appears at approximately $2900 \mathrm{~cm}^{-1}$ (Figure 5c).

\section{Cytotoxicity}

Cytotoxicity pattern was variable among the synthesized ZNPs and revealed a concentration

dependent decrease in the viability of Vero cells along with increasing the concentration of the tested nanoparticles. OA-ZNPs was the most cytotoxic followed by H-ZNPs, where the safe concentration that maintained the cellular viability was $0.01 \& 0.1 \mathrm{mg} / \mathrm{mL}$; respectively. On the other hand, C-ZNPs showed the least cytotoxicity where it was completely non-toxic at a concentration of $0.24 \mathrm{mg} / \mathrm{mL}$ (Figure 6).

\section{Effect on early stages of viral infection (cell receptor occupation potential)}

Table 1: Effect of ZNPs on early stages of viral infection in ZNPs pretreated cells.

\begin{tabular}{|l|l|l|}
\hline Zn nanoparticles & $\begin{array}{l}\text { Depletion in viral } \\
\text { titer }\end{array}$ & $\begin{array}{l}\text { Percentage } \\
\text { reduction in } \\
\text { viral titer }\end{array}$ \\
\hline OA-ZNPs & $\log (10) / 0.1 \mathrm{~mL} \pm \mathrm{SD}$ & \\
\hline C-ZNPS & $0.5 \pm 0.08$ & 7.7 \\
\hline H-ZNPs & 0 & 0 \\
\hline
\end{tabular}
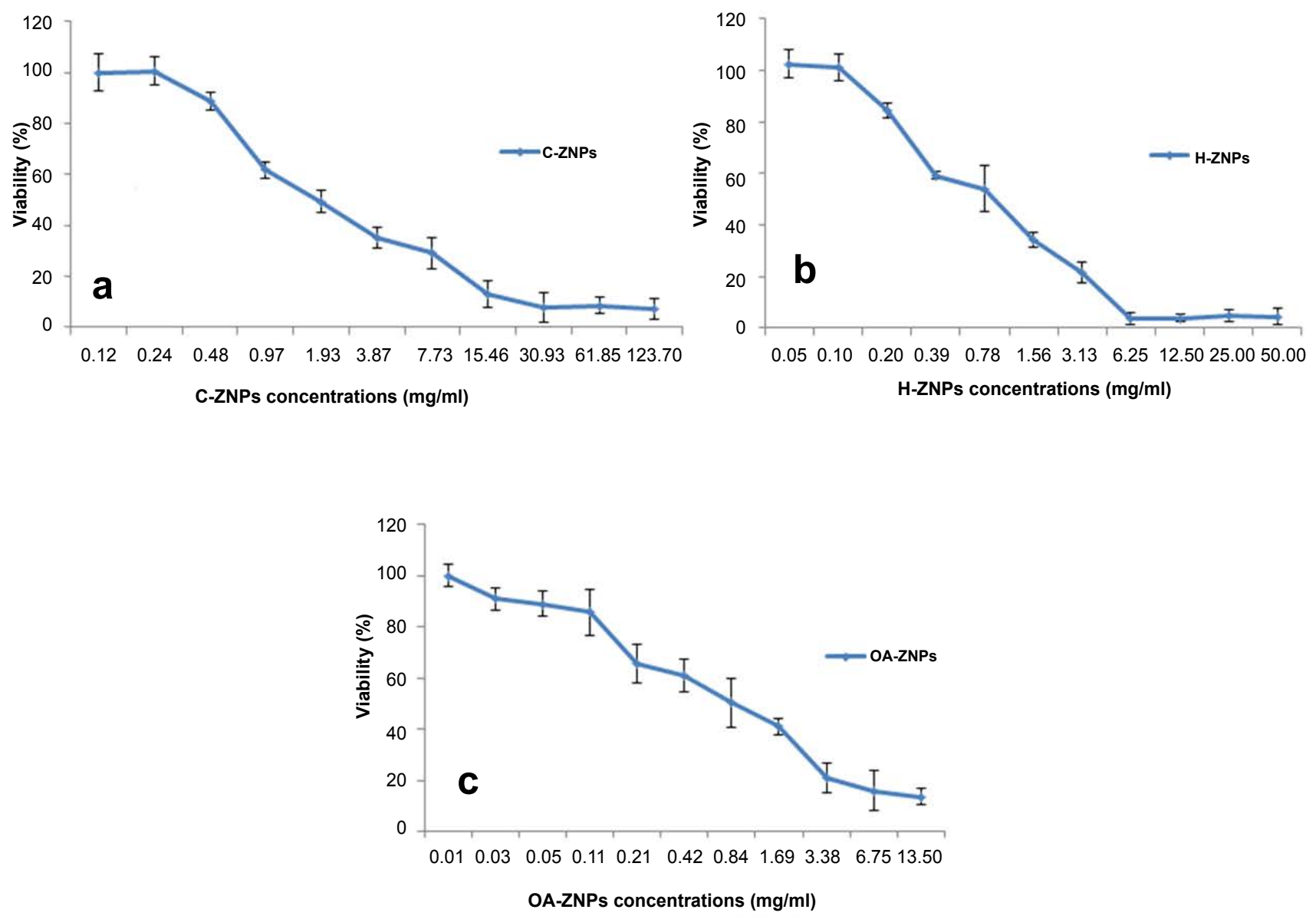

Figure 6: Cytotoxicity assay of H-ZNP (a) C-ZNP (b) OA-ZNP (c) Against Vero cells. The assay was based on the amount of active lactate dehydrogenase released from residual viable cells. Recorded values were the mean of independent triplicates \pm standard deviation. The results reveal a concentration dependent cytotoxicity. 
Table 2: Viral inhibitory potential of ZNPs at different time interval.

\section{Percentage reduction in viral titer \pm SD}

\begin{tabular}{|c|c|c|c|c|}
\hline Zn nanoparticles & Time post & & & \\
\hline $24 \mathrm{hrs}$ & $4 \mathrm{hrs}$ & $2 \mathrm{hrs}$ & $1 \mathrm{hr}$ & \\
\hline $15 \pm 0.8$ & $9.5 \pm 0.9$ & $7.7 \pm 1.1$ & $0.4 \pm 0.02$ & OA-ZNPs \\
\hline $100 \pm 7.7$ & $71 \pm 6.4$ & $30 \pm 1.9$ & - & C-ZNPs \\
\hline $100 \pm 8.6$ & $65.5 \pm 5.1$ & $19.4 \pm 3.7$ & $11.7 \pm 0.3$ & H-ZNPs \\
\hline
\end{tabular}

Evaluation of the antiviral activity of ZNPs revealed that C-ZNPs didn't show any inhibitory effect on the early stages of viral infection, while OA-ZNPs and H-ZNPs pretreated cells resulted in minor reduction in the viral titer in the order of $7.7 \%$ and $12.9 \%$, respectively $(p<0.05)$ (Table 1$)$.

\section{Viral inhibitory potential (Viral neutralization potential)}

Assessment of the inhibitory activity of ZNPs on HSV-1 at different time interval $(1,2,4$ \& 24 h) indicated that OA-ZNPs $(0.01 \mathrm{mg} / \mathrm{mL})$ exhibited the least inhibitory effect on HSV-1 as compared to $\mathrm{H}$-ZNPs and C-ZNPs, where the percentage reduction in the viral titer in case of OA-ZNPs was $0.4 \%$, $7.7 \%, 9.5 \%$ and $15 \%$, respectively. On the other hand, H-ZNPs $(0.01 \mathrm{mg} / \mathrm{mL})$ showed a promising time dependent significant reduction in the viral infectivity titer in the order of $11.7 \%, 19.4 \%$ and $65.5 \%$ post 1,2 and $4 \mathrm{~h}$ of viral treatment. Despite that treating the virus with C-ZNPs for a period of $1 \mathrm{~h}$ wasn't accompanied by reduction in the virus infectivity titer, prolonged contact time between HSV-1 and C-ZNPs $(0.24 \mathrm{mg} / \mathrm{mL})$ resulted in the highest reduction in the viral infectivity by $30 \%$ and $71 \%$ post 2 and $4 \mathrm{~h}$ of treatment, respectively ( $\mathrm{p}$ $<0.05)$. In the meantime, complete inactivation of HSV-1 (with an initial viral titre of $10^{6}$ cell culture infectious dose $50 / 0.1 \mathrm{~mL}$ ) was achieved post $24 \mathrm{~h}$ of ZNPs treatment in case of both H-ZNPs and C-ZNPs as shown in Table $2(p<0.01)$.

\section{Discussion}

Viral diseases are considered one of the most serious public health concerns which are associated with social and economic consequences. The development of a potential antiviral agent that successfully targets the virus without exerting a hazard on the viability of the host cell remains a challenge. Acyclovir and other related compounds are the current gold standards for treatment of HSV infections. They are virustatic agents that are effective during early stages of infection [28]. Unfortunately, acyclovir resistant strains are increasing especially in immune-compromised patients due to the prolonged time required for HSV treatment in these patients as compared to immune-competent ones $[6,36,37]$.

New strategies for management of HSV are emerging such as the use of the DNA helicase/ primase (H/P) complex inhibitors. Pritelivir (BAY 57-1293) is a potent helicase primase inhibitor which is more effective against HSV-1 than famciclovir $[38,39]$. Another strategy rely on using a novel nucleoside analogue such as valomaciclovir stearate (EPB-348), where it exhibited an in vitro activity aganist HSV-1 [39]. These small molecule compounds are still under costly and long clinical trials [39]. In addition, most of these drugs still require immense pharmacokinetic adjustment [40].

The use of NPs represents valuable alternative. Metal NPs can offer remarkable achievements in the field of treatment of viral infections due to their distinctive chemical and physical properties as well as their high surface area to volume ratio which allow them to interact with variable targets. Such approach has a lower probability for the development of resistance compared to small molecule approach [41]. The chemical synthesis of NPs can be considered simple when compared to the synthesis of small molecule drugs. In addition, their surface physico-chemical properties can be easily tailored for optimizing their interaction pattern with the biological targets accordingly a great benefit could be obtained from the core material of the NPs in addition to the ligands of their shells $[42,43]$.

This is especially important because the interaction pattern between viruses and the host cell involves multiple receptors and ligands. Accordingly, the development of compounds that inhibit viral attachment to the host cell through mimicking or targeting viral receptors on the host cell or using compounds that binds to the virion itself is gaining interest $[44,45]$. This is a special privilege for NPs based therapeutics where their 
surface functionalization is easily achievable [42].

ZNPs were previously reported active against HSV. Mishra, et al. reported the virostatic potential of zinc micro-nano filopodia-like ZnO against HSV-1 at a concentration of $0.1 \mathrm{mg} / \mathrm{mL}[22,23]$. Following them Antoine, et al. proved that zinc nano tetrapods have a similar antiviral activity against HSV-2 as well [24].

Whereas the recruited zinc structure was synthesized by an energy consuming procedures while the virostatic potential was only attributed to inhibiting the electrostatic interaction between the virus and HS of the host cell [22,24], The simpler $\mathrm{H}$-ZNP were designed to target the cationic viral glycoproteins in order to neutralize HSV-1. They were successfully synthesized using a simple coprecipitation method which produced a high yield at room temperature. The design was based on the assumption that hydroxyl group rich structure of H-ZNPs may mask the positivity of the viral glycoprotein and reduces its inherent ability to interact with the cellular HS [46]. That was in consistence with the recorded ability of these particles to counteract the viral infectivity potential when they were incubated with the virus even for a short period of time.

The recorded lower reduction in the viral titer in case of H-ZNP pretreated cells compared to their inhibitory potentials when the virus was directly treated with NPs supports that the effect of these particles is more directed towards neutralizing the virus rather than to the viral targets on the host cell. It is worth pointing out that the H-ZNP produced similar results (even in the effective concentration) to those zinc micro-nano filopodialike $\mathrm{ZnO}$ synthesized by an energy consuming procedure $[22,24]$.

The electrostatic interaction between the virus and the target cell is subsequently followed by viral fusion into cell membrane which is mediated by the hydrophobic residues in the viral glycoprotein's fusion peptides (example is the $\mathrm{gH}$ ) $[17,18]$. OA-ZNPs were proposed to interfere with such hydrophobic interaction due to their hydrophobic coat. However, they exhibited a reduced potential compared to that of H-ZNP. The apparent low inhibitory activity in case of OA-ZNPs pretreated cells $(7.7 \%)$ as well as its ability to only inactivate $15 \%$ of the virus post $24 \mathrm{hr}$ of viral treatment may be related to their high toxicity on Vero cell and consequently to the lower concentration that was applied in the present study $(0.01 \mathrm{mg} / \mathrm{ml})$ compared to $0.1 \& 0.24 \mathrm{mg} / \mathrm{mL}$ in case of H-ZNPs and C-ZNPs, respectively.

Chitosan was reported to exhibit indirect viral inhibitory potentials as attributed to its ability to elicit innate immunity of cells [47]. Being a cationic molecule, it is able to interact with the outer surface of cell membrane which may trigger immune response [48]. Additionally, although chitosan might not have a virudical activity on its own especially against positively charged viral envelope glycoproteins, its conjugation with viral reactive structures proved successful $[49,50]$. Based on these desirable properties of chitosan composites, C-ZNPs were synthesized composed of ZNP embedded in the chitosan matrix [50].

No inhibitory potential was observed on pretreatment of the cells with C-ZNP while a reduction in the viral infectivity potential was observed post incubating the C-ZNPs with HSV-1 for different time intervals. Such effect may be exerted by the physical barrier the composite offers which may entrap the viral particles, thus preventing their adsorption to the host cell. The failure of C-ZNPs to exhibit any viral inactivation potential post one hour of viral treatment may be related to the fact that the physical entrapment of the virus in the composite requires a time to be achieved. Despite that both C-ZNPs and H-ZNPs completely inactivated the virus post $24 \mathrm{~h}$ of treatment, but C-ZNPs showed greater inactivation kinetics compared to that in case of $\mathrm{H}$-ZNPs post $2 \& 4 \mathrm{~h}$ of incubation with the tested NPs.

In summary, H-ZNPs and OA-ZNPs resulted in reduction in the viral infectivity titer in the order of $12.9 \%$ and $7.7 \%$; respectively in nanoparticles pretreated cells whereas C-ZNPs were ineffective. Incubating HSV-1 with H-ZNPs and C-ZNPs showed a greater time dependent inhibition and completely inactivated the virus within a period of $24 \mathrm{~h}$ while OA-ZNPs exhibited the lowest reduction in the viral titer by a value of $15 \%$.

To the best of our knowledge, this is the first study that evaluated the effect of electrostatic, physical and hydrophobic interaction of ZNPs on the infectivity potential of HSV-1 in terms of the effect of the particles on the early stages of viral entry into the cell compared to the ability of the prepared nanoparticles to neutralize the virus. 


\section{Conclusion}

The present study evaluated the anti-viral potential of ZNPs. This was achieved by their successful surface functionalization with polyhydroxy group (H-ZNP), chitosan (C-ZNP) and oleic acid (OA-ZNP) for interfering electrostatically, physically and hydrophobically with HSV-1 entry into the cell either via targeting cellular receptors or neutralizing the virus itself.

The H-ZNPs exhibited the highest antiviral potential in nanoparticles pretreated cells where the percentage reduction in viral titer was $12.9 \%$. This was followed by $7.7 \%$ reduction in case of OA-ZNPs treatment. The C-ZNPs was totally ineffective in this respect. On the other hand, the viral neutralizing potential of C-ZNPs was the most potent however its effect was delayed compared to other forms of ZNPs. The H-ZNPs showed an earlier onset (12\% reduction HSV-1 in infectivity after 1 hour) but weaker viral neutralizing potential as compared to that of C-ZNPs. Both the C-ZNPs and the H-ZNPs were able to completely neutralize the virus after 24 hours. The OA-ZNP was the least effective with $15 \%$ inhibition even after 24 hours.

Current results suggested that the surface modified ZNPs can potentially modify the infectivity potential of HSV-1 by exerting their effect on viral particles rather than through interfering with cellular targets. Where targeting the electrostatic interaction between HSV-1 and the host cells by $\mathrm{H}$-ZNP exhibited the highest and the fastest effect on the viral infectivity titer followed by the C-ZNP which may exert its function via entrapping the virus and preventing its access to cell surface. It is worth noting that C-ZNP may represent a potential alternative to counteract the viral infectivity but is accompanied by a delay in the initiation of its effect. On the other hand, targeting the hydrophobic interaction represent the least effect. In conclusion the cell safe concentrations of H-ZNP and C-ZNP were able to neutralize the HSV-1 and reduce its infection in host cells.

\section{Conflict of Interest}

None.

\section{Funding Section}

No funding was received for this research.

\section{References}

1. Vanjeri S, Galwankar S (2012) State of the globe:
Detecting HSV in clinical setting. J Glob Infect Dis 4: $139-140$.

2. World Health Organisation (2016) Globally, an estimated two-thirds of the population under 50 are infected with herpes simplex virus type 1.

3. Arduino $P$, Porter $S$ (2006) Oral and perioral herpes simplex virus type 1 (HSV-1) infection: Review of its management. Oral Dis 12: 254-270.

4. Lafferty WE, Downey L, Celum C, Wald A (2000) Herpes simplex virus type 1 as a cause of genital herpes: impact on surveillance and prevention. J Infect Dis 181: 1454-1457.

5. Roberts CM, Pfister JR, Spear SJ (2003) Increasing proportion of herpes simplex virus type 1 as a cause of genital herpes infection in college students. Sex Transm Dis 30: 797-800.

6. Jiang YC, Feng H, Lin YC, Guo XR (2016) New strategies against drug resistance to herpes simplex virus. Int J Oral Sci 8: 1-6.

7. Whitley RJ, Roizman B (2001) Herpes simplex virus infections. Lancet 357: 1513-1518.

8. Trybala E, Liljeqvist JA, Svennerholm B, Bergström $T$ (2000) Herpes simplex virus types 1 and 2 differ in their interaction with heparan sulfate. J Virol 74: 9106-9114.

9. Weitz JI (2003) Heparan sulfate: Antithrombotic or not? J Clin Invest 111: 952-954.

10. Sarrazin S, Lamanna WC, Esko JD (2011) Heparan sulfate proteoglycans. Cold Spring Harb Perspect Biol 3: a004952.

11. Rabenstein DL (2002) Heparin and heparan sulfate: Structure and function. Nat Prod Rep 19: 312-331.

12. Davis S, Anak D, Parish CR (2013) Heparan sulfate: A ubiquitous glycosaminoglycan with multiple roles in immunity. Front Immunol 4: 470.

13. Lin X (2004) Functions of heparan sulfate proteoglycans in cell signaling during development. Development 131: 6009-6021.

14. Shukla D, Liu J, Blaiklock $P$, Shworak NW, Bai X, et al. (1999) A novel role for 3-O-sulfated heparan sulfate in herpes simplex virus 1 entry. Cell 99: 13-22.

15. Mårdberg K, Trybala E, Glorioso JC, Bergström T (2001) Mutational analysis of the major heparan sulfate-binding domain of herpes simplex virus type 1 glycoprotein C. J Gen Virol 82: 1941-1950.

16. Trybala E, Bergström T, Svennerholm B, Jeansson S, Glorioso JC, et al. (1994) Localization of a functional site on Herpes simplex virus type 1 glycoprotein C 
involved in binding to cell surface heparan sulphate. J Gen Virol 75: 743-752.

17. Galdiero S, Falanga A, Vitiello M, Raiola L, Fattorusso $R$, et al. (2008) Analysis of a membrane interacting region of herpes simplex virus type 1 glycoprotein H. J Biol Chem 283: 29993-30009.

18. Galdiero S, Falanga A, Vitiello G, Vitiello M, Pedone C, et al. (2010) Role of membranotropic sequences from herpes simplex virus type I glycoproteins B and $\mathrm{H}$ in the fusion process. Biochim Biophys Acta 1798: 579-591.

19. Read SA, Parnell G, Booth D, Douglas MW, George J, et al. (2018) The antiviral role of zinc and metallothioneins in hepatitis C infection. J Viral Hepat 25: 491-501.

20. Wei Z, Burwinkel M, Palissa C, Ephraim E, Schmidt MF (2012) Antiviral activity of zinc salts against transmissible gastroenteritis virus in vitro. Vet Microbiol 160: 468-472.

21. Arens M, Travis S (2000) Zinc salts inactivate clinical isolates of herpes simplex virus in vitro. J Clin Microbiol 38: 1758-1762.

22. Mishra YK, Adelung R, Röhl C, Shukla D, Spors F, et al. (2011) Virostatic potential of micro-nano filopodialike $\mathrm{ZnO}$ structures against herpes simplex virus-1. Antiviral Res 92: 305-312.

23. Duggal N, Jaishankar D, Yadavalli T, Hadigal S, Mishra YK, et al. (2017) Zinc oxide tetrapods inhibit herpes simplex virus infection of cultured corneas. Mol Vis 23: 26-38.

24. Antoine TE, Mishra YK, Trigilio J, Tiwari V, Adelung $R$, et al. (2012) Prophylactic, therapeutic and neutralizing effects of zinc oxide tetrapod structures against herpes simplex virus type- 2 infection. Antiviral Res 96: 363-375.

25. Singh RC, Onkar Singh, Manmeet Pal Singh, Paramdeep Singh Chandi (2008) Synthesis of zinc oxide nanorods and nanoparticles by chemical route and their comparative study as ethanol sensors. Sensors and Actuators B: Chemical 135: 352-357.

26. Leila D, López-González Mar, Belhadj Fatima, Naitbouda Abddelyamine, Benosmane Ali, et al. (2018) Effect of polyethylene glycol and propyltrimethoxysilane on structural and optical properties of zinc oxide nanoparticles synthesized by sol-gel process. Journal of Theoretical and Applied Physics 12: 159-167.

27. McAteer JA, Davis J (1994) Basic cell culture technique and the maintenance of cell lines. Basic cell culture: A practical approach 1994: 109-143.
28. Houston DM, Bugert JJ, Denyer SP, Heard CM (2017) Potentiated virucidal activity of pomegranate rind extract (PRE) and punicalagin against Herpes simplex virus (HSV) when co-administered with zinc (II) ions, and antiviral activity of PRE against HSV and aciclovir-resistant HSV. PloS one 12: e0179291.

29. Patravale V, Dandekar, Jain R (2012) Nanoparticulate drug delivery: Perspectives on the transition from laboratory to market. Elsevier.

30. Chung DH, Golden JE, Adcock RS, Schroeder CE, Chu YK, et al. (2016) Discovery of a broad-spectrum antiviral compound that inhibits pyrimidine biosynthesis and establishes a type 1 interferonindependent antiviral state. Antimicrob Agents Chemother 60: 4552-4562.

31. Ramadan R, Mohamed A, ABD Mohamed (2009) Evaluation of antiviral activity of honeybee venom on DNA and RNA virus models. Egypt Acad J Biolog Sci 2: 247-258.

32. Hong R, Tingting Pan, Jianzhong Qian, Hongzhong Lib (2006) Synthesis and surface modification of $\mathrm{ZnO}$ nanoparticles. Chemical Engineering Journal 119: 71-81.

33. Liufu S, Xiao H, Li Y (2004) Investigation of PEG adsorption on the surface of zinc oxide nanoparticles. Powder Technology 145: 20-24.

34. Hong RY, Li JH, Chen LL, Liu DQ, Li HZ, et al. (2009) Synthesis, surface modification and photocatalytic property of ZnO nanoparticles. Powder Technology 189: 426-432.

35. Mahdavi M, Ahmad MB, Haron MJ, Namvar F, Nadi B, et al. (2013) Synthesis, surface modification and characterisation of biocompatible magnetic iron oxide nanoparticles for biomedical applications. Molecules 18: 7533-7548.

36. Kakiuchi S, Tsuji M, Nishimura $H$, Yoshikawa $T$, Wang L, et al. (2017) Association of the emergence of acyclovir-resistant herpes simplex virus type 1 with prognosis in hematopoietic stem cell transplantation patients. J Infect Dis 215: 865-873.

37. Frobert E, Burrel S, Ducastelle-Lepretre S, Billaud G, Ader F, et al. (2014) Resistance of herpes simplex viruses to acyclovir: An update from a ten-year survey in France. Antiviral Res 111: 36-41.

38. Biswas S, Jennens L, Field HJ (2007) The helicase primase inhibitor, BAY 57-1293 shows potent therapeutic antiviral activity superior to famciclovir in $B A L B / c$ mice infected with herpes simplex virus type 1. Antiviral Res 75: 30-35. 
39. Birkmann A, Zimmermann H (2017) Drugs in development for herpes simplex and varicella zoster virus. Clin Pharmacol Ther 102: 30-32.

40. Kreemerova M (2017) Amino acid ester prodrugs of nucleoside and nucleotide antivirals. Mini Rev Med Chem 17: 818-833.

41. Galdiero S, Falanga A, Vitiello M, Cantisani M, Marra $V$, et al. (2011) Silver nanoparticles as potential antiviral agents. Molecules 16: 8894-8918.

42. Singh L, Hendrik G Kruger, Glenn EM Maguire, Thavendran Govender, Raveen Parboosing (2017) The role of nanotechnology in the treatment of viral infections. Ther Adv Infect Dis 4: 105-131.

43. Ravishankar Rai V, Jamuna Bai A (2011) Nanoparticles and their potential application as antimicrobials. Mysore: Formatex.

44. Spjut S, Qian W, Bauer J, Storm R, Frängsmyr L, et al. (2011) A potent trivalent sialic acid inhibitor of adenovirus type 37 infection of human corneal cells. Angew Chem Int Ed Engl 50: 6519-6521.
45. Marsh M, Helenius A (2006) Virus entry: Open sesame. Cell 124: 729-740.

46. Yang XX, Li CM, Huang CZ (2016) Curcumin modified silver nanoparticles for highly efficient inhibition of respiratory syncytial virus infection. Nanoscale 8: 3040-3048.

47. Iriti M, Varoni EM (2015) Chitosan-induced antiviral activity and innate immunity in plants. Environ Sci Pollut Res Int 22: 2935-2944.

48. Hamel L-P, Beaudoin N (2010) Chitooligosaccharide sensing and downstream signaling: Contrasted outcomes in pathogenic and beneficial plantmicrobe interactions. Planta 232: 787-806.

49. Cheng S, Zhao H, Xu Y, Yang Y, Lv X, et al. (2014) Inhibition of influenza virus infection with chitosansialyloligosaccharides ionic complex. Carbohydr Polym 107: 132-137.

50. Mori Y, Ono T, Miyahira Y, Nguyen VQ, Matsui T, et al. (2013) Antiviral activity of silver nanoparticle/ chitosan composites against H1N1 influenza A virus. Nanoscale Res Lett 8: 93.

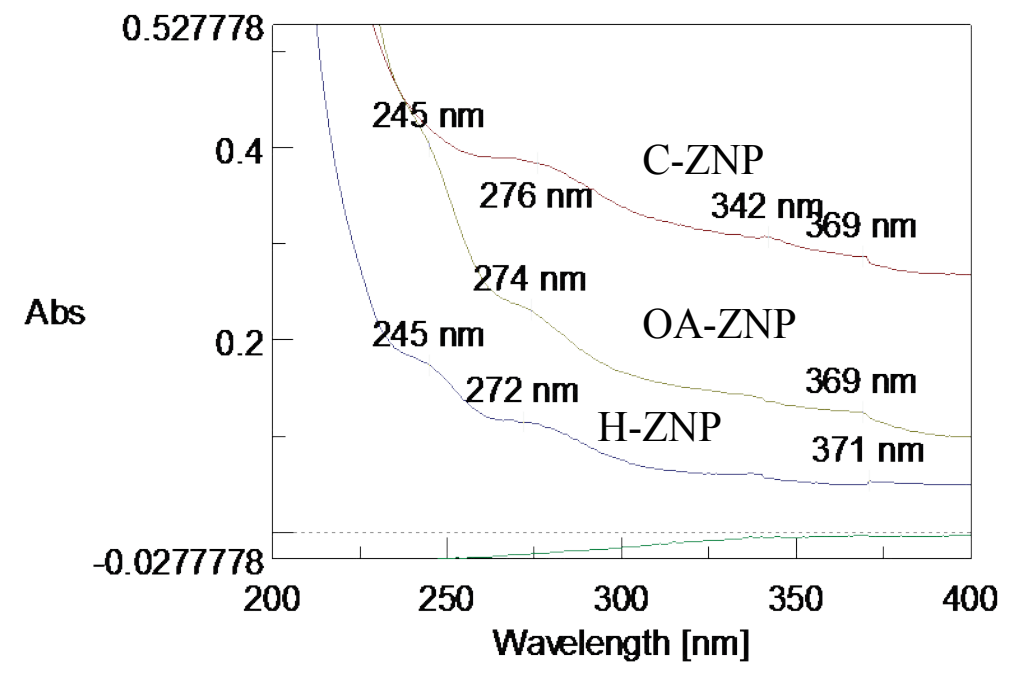

Supplementary Material: UV spectrum of HZNP; OA-ZNP and C-ZNP. 